\title{
OPINIÓN
}

\section{Los isótopos estables como potenciales indicadores de deforestación en cuencas hidrográficas}

\author{
Stable isotopes as potential deforestation indicators in watersheds
}

\author{
Iván Arismendia, b \\ aUniversidad Austral de Chile, Facultad de Ciencias Forestales, Escuela de Graduados. \\ bNúcleo Milenio FORECOS, casilla 567 Valdivia, Chile, fono-fax: 56-63-293418, ivanarismendi@uach.cl
}

\begin{abstract}
SUMMARY
Deforestation increases maximum stream flows, and soil erosion. This article proposes the use of stable isotopes to better understand the effect of the forest on the hydrological cycle. The isotopic fractionation is different depending on the type and density of the forest, which provides a specific rate between key isotopes in the sediments and key isotopes from runoff. This proportion can detect changes between watersheds with forests and others with intervention, thus this value can be used as an indicator of deforestation. Paired watersheds should be used to begin such studies using stable isotopes $\mathrm{H}, \mathrm{O}, \mathrm{N}$ and $\mathrm{C}$ from both water and sediments.
\end{abstract}

Key words: forest runoff, isotopic fractionation, hydrological cycle.

\section{RESUMEN}

La deforestación incrementa los caudales máximos provocando además la pérdida de suelo por erosión. Este trabajo propone el uso de isótopos estables para entender mejor el efecto del bosque en el ciclo hidrológico. Se plantea un fraccionamiento isotópico diferenciado según el tipo y densidad del bosque, que permitiría obtener una razón distinta entre isótopos de elementos claves, tanto en sedimentos como en agua de los cauces. La detección de cambios en dicha razón isotópica entre cuencas cubiertas por bosque con diferente grado de intervención puede utilizarse como indicador de deforestación. Se propone comenzar estudios a través de experimentos en cuencas pareadas, utilizando isótopos estables de hidrógeno, oxígeno, nitrógeno y carbono.

Palabras clave: caudales, bosque, fraccionamiento isotópico, ciclo hidrológico.

\section{INTRODUCCIÓN}

En todo el mundo, los procesos de deforestación han creado graves problemas en cuanto a fragmentación y disminución de la biodiversidad (Wright y Flecker 2004, Cayuela et al. 2006, Echeverría et al. 2006), pérdida de suelos por erosión (Ward y Trimble 2004, Collard y Zammit 2006) y cambios en la distribución y monto de la escorrentía superficial (Croke et al. 2004, Brown et al. 2005). Este último caso ha generado una intensa discusión sobre cuál es el real impacto que provocan los bosques en el régimen hidrológico (Calder 1998, Andreassian 2004, Brown et al. 2005). A nivel de cuenca, se sabe que los bosques tienen influencia en el balance hídrico, ya que consumen agua en mayor proporción que otros tipos de vegetación; sin embargo, no se tiene suficiente información sobre las consecuencias de la composición etaria y densidad del bosque en dicho balance (Andreassian 2004). Lo anterior ha generado la necesidad de explorar nuevas herramientas para entender mejor estas consecuencias.

Una de las técnicas que se ha desarrollado en los últimos 50 años es el uso de isótopos estables (IE) de distintos elementos tales como carbono, oxígeno, hidrógeno y nitrógeno. Esta técnica ha sido de gran utilidad en el seguimiento de procesos ecológicos y fisiológicos (Squeo y Ehleringer 2004). Ello, debido a que los IE tienen un relativo bajo costo (Squeo y Ehleringer 2004), no decaen radiactivamente (Kendall y Caldwell 1998, De Vries 2000), y pueden mantener en el tiempo la razón entre el isótopo más liviano y el más pesado (De Vries 2000, White 2005).

Al ocurrir un incremento en la abundancia de un isótopo del mismo elemento relativo a otro, el proceso que genera dicha acción se denomina fraccionamiento isotópico (Kendall y Caldwell 1998, White 2005), el cual es clave para entender el uso de IE como indicadores de deforestación. Ello, debido a que el fraccionamiento va 
a ser diferente de acuerdo al tipo y densidad de bosque, lo que se va a reflejar en un cuociente distinto entre los isótopos del mismo elemento durante su recorrido en el ciclo hidrológico. El objetivo del presente trabajo de revisión es analizar la factibilidad del uso de IE como indicadores de deforestación, a través del seguimiento de la razón isotópica de elementos claves en algunos componentes del ciclo hídrico.

\section{LA DEFORESTACIÓN Y SU INFLUENCIA EN LOS CAUDALES}

Andreassian (2004), en una exhaustiva revisión histórica de la literatura científica referida al impacto de los bosques en los caudales, concluye que la tala de árboles genera en una primera etapa un incremento en la producción de agua, lo que va seguido de una segunda etapa de disminución en ella. Esta primera etapa, de mayor escorrentía superficial, concuerda con experiencias realizadas en el centro sur de Chile, donde se estima que cuencas con diferente uso de suelo aumentaron su caudal de verano en condición de menor cobertura vegetal (Iroumé et al. 2005). Por su parte, en situación postala rasa, el caudal anual creció más del doble, y en un tercio los caudales máximos (Iroumé et al. 2006). La segunda etapa de disminución en los caudales mencionada por Andreassian (2004), se atribuye al establecimiento y crecimiento del nuevo bosque, produciéndose una mayor intercepción de las precipitaciones en el dosel y un mayor consumo producto de la evapotranspiración. Además, se reconoce esta etapa como de duración muy variable, debido principalmente a las condiciones de clima, suelo y tipo de bosque en cada caso particular (Brown et al. 2005). Por ejemplo, un estudio realizado en Australia muestra la existencia de un claro efecto de reducción en los caudales (31-44\%), luego de sustituir praderas y matorrales con plantaciones de pino y eucalipto (Farley et al. 2005). De igual forma, en Chile se ha encontrado que dichas plantaciones de especies exóticas provocan una importante reducción del agua disponible en el suelo (Huber et al. 1998, Huber y Trecaman 2004).

Otra consecuencia de los procesos de deforestación es la pérdida de suelo por erosión (Ward y Trimble 2004, Collard y Zammit 2006). Particularmente en Chile, se ha estudiado que los volúmenes de sedimentos que llegan a los cauces están vinculados principalmente a erosión superficial, por lo que su transporte es en forma de suspensión (Iroumé 1990). En cuencas sin vegetación y con uso agrícola-ganadero se ha encontrado una exportación tanto de fósforo como de nitrógeno total mucho mayor que en cuencas cubiertas por bosques nativos (Oyarzún et al. 1997, Oyarzún y Huber 2003, Alfaro et al. 2005). Adicionalmente, Oyarzún y Peña (1995) indican que en la Cordillera de la Costa (IX Región), después de la cosecha de una plantación de Pinus radiata D. Don, durante el primer año, la pérdida de suelo fue 44,3 veces mayor a lo ocurrido en suelos no perturbados.

\section{ISÓTOPOS ESTABLES COMO INDICADORES DE DEFORESTACIÓN}

Debido a que las consecuencias de la deforestación se reflejan como cambios en escorrentías superficiales y exportación de sedimentos, es necesario considerar como claves los IE de elementos constituyentes de los caudales. De este modo, y para entender la distribución isotópica de dichos constituyentes, es importante conocer primero el origen de la fuente acuífera (aguas subterráneas, nieve y/o precipitaciones), la cual está influenciada por el fraccionamiento que existió durante su proceso de formación, así como por la propia variabilidad edafoclimática y geográfica de cada sitio (Poage y Chamberlain 2001, Clay et al. 2004, Berdea et al. 2005). Por este motivo, los IE de hidrógeno y oxígeno son considerados excelentes trazadores, ya que constituyen las moléculas de agua en sí mismas, y contienen las señales distintivas de las diferentes masas de agua que componen los caudales (Gremillion et al. 2000). Por ejemplo, Gibson et al. (2002), a través de IE de hidrógeno y oxígeno, encontraron que en cuencas presentes en climas húmedos, las precipitaciones son el primer signo visible en los caudales de los ríos. A su vez, Clay et al. (2004), en un estudio desarrollado en humedales del Reino Unido, hallaron que las aguas subterráneas son proporcionalmente más importantes como fuente acuífera; sin embargo, bajo ciertas condiciones climáticas, el aporte de precipitación y flujo de arroyos afluentes puede ser relevante. Por otra parte, varios estudios muestran que luego de un evento de precipitación, la evaporación enriquece las hojas en isótopos de agua más pesados, debido a que los isótopos livianos se evaporan más rápidamente (Cooper y DeNiro 1989, Roden y Ehleringer 1999, White 2005). Ello sugiere que los procesos de condensación y evaporación sucesiva del agua dentro del bosque van a dar una señal isotópica propia a la masa de agua que va a drenar dicha cuenca. Por ejemplo, Liu et al. (2006), utilizando IE de hidrógeno en modelos de balance de masas, miden la contribución efectiva de los diferentes tributarios hacia las grandes cuencas, identificando cada señal de acuerdo a su cobertura vegetal. Lo anterior sugiere, entonces, que en la ocurrencia de procesos de deforestación y tala rasa, los cambios que se generen en las escorrentías superficiales van a modificar la proporcionalidad de masas de agua constituyentes del caudal y, por ende, su razón isotópica.

Al existir una estrecha interacción entre la vegetación, el agua que llega al suelo, aguas subterráneas y caudal de los ríos (Gremillion y Wanielista 2000, Henderson-Sellers et al. 2004), es probable que parte de la escorrentía superficial de una cuenca contenga las señales isotópicas 
del suelo que atraviese. Ashkenas et al. (2004) señalan que en arroyos localizados dentro de bosques primarios imperturbados en la costa pacífica de Estados Unidos, el consumo y retención de nitrógeno es muy eficiente, por lo que no se exportaría hacia los cauces. Además, el patrón isotópico de nitrógeno y carbono sería dependiente de la profundidad de la materia orgánica (Billings y Richter 2006). Como la deforestación y conversión hacia uso agrícola va a causar compactación y erosión del suelo (Hatchell et al. 1970, Ward y Trimble 2004, Collard y Zammit 2006), dicha señal isotópica se trasladará en algún momento hacia los cauces. Por ejemplo, en el sur de Chile se ha estudiado que cuencas donde se ha intensificado el uso de fertilizantes más del $70 \%$ del $\mathrm{N}$ total en el agua es inorgánico (Oyarzún y Huber 2003). Lo anterior sugiere que cambios en la proporción de nitrógeno y carbono en el suelo pueden detectarse como modificaciones en la razón entre IE de dichos elementos en el agua y sedimentos de la red de drenaje. De hecho, Ehleringer et al. (2002) indican que la composición isotópica asociada con deforestación puede cambiar estacional e interanualmente las señales isotópicas de carbono. A su vez, Phillips et al. (2003), presentando cambios en series de tiempo de la razón isotópica en solutos presentes en el agua, señalan la potencialidad de los IE como identificadores de fuentes de contaminación. Finalmente, otros autores han explorado la relación entre IE de nitrógeno y oxígeno en los nitratos, los cuales, de acuerdo a su proporcionalidad, indicarían diferentes señales de origen y uso de suelo agrícola y ganadero (Battaglin et al. 2001, Lake et al. 2001, Chang et al. 2002).

De acuerdo a los antecedentes expuestos en el presente trabajo, se concluye que sin duda existe un gran potencial en los IE como indicadores de deforestación. Se desprende además que se debe comenzar su estudio utilizando oxígeno, hidrógeno, carbono y nitrógeno como elementos claves, tanto en sedimentos como en el agua de escorrentía superficial y precipitaciones. Se recomienda considerar un diseño experimental de cuencas pareadas con calibración previa, según lo propuesto por Andreassian (2004). De esta forma, se pueden construir relaciones de los IE de cada elemento en un sitio control y en uno a intervenir. Luego, las diferencias de dichas relaciones entre sitios se pueden considerar como proporcionales a la intervención realizada. Finalmente, se debe tener en cuenta la variabilidad en la geología, suelos y clima, por lo que las investigaciones deben utilizar cada zona edafoclimática como una unidad experimental independiente. Además, es necesario analizar el efecto adicional que tienen fenómenos climáticos remotos, tales como El Niño, los cuales actúan como desencadenantes de cambios adicionales en las señales isotópicas, sobre todo en las precipitaciones (Hoffmann 2003, Vuille y Werner 2005).

\section{REFERENCIAS}

Alfaro M, F Salazar, S Iraira, N Teuber, L Ramírez. 2005. Nitrogen runoff and leaching losses in beef production systems under two different stocking rates in Southern Chile. Gayana Bot. 62(2): 130-138.

Andreassian V. 2004. Waters and forests: from historical controversy to scientific debate. Journal of Hydrology 291: 1-27.

Ashkenas L, S Johnson, G Stan, J Tank, W Wollheim. 2004. A stable isotopes tracer study of nitrogen uptake and transformation in an old-growth forest stream. Ecology 85(6): 1725-1739.

Battaglin W, C Kendall, C Chang, S Silva, D Campbell. 2001. Chemical and isotopic evidence of nitrogen transformation in the Mississippi River, 1997-98. Hydrological Processes 15: $1285-1300$.

Billings S, D Richter. 2006. Changes in stable isotopic signatures of soil nitrogen and carbon during 40 years of forest development. Oecologia 148: 325-333.

Berdea P, S Cuna, O Cozar, G Muresan. 2005. Application of stable isotopes $\left({ }^{18} \mathrm{O}\right.$ and $\left.\mathrm{D}\right)$ to study the provenience of mineral waters from some locations of Romania. Rom. Journ. Phys. 50(7-8): 685-690.

Brown A, I Zhang, T McMahon, A Western, R Vertessy. 2005. A review of paired catchment studies for determining changes in water yield resulting from alterations in vegetation. Journal of Hydrology 310: 28-61.

Calder I. 1998. Water-resource and land-use issues. SWIM Paper 3. Colombo, Sri Lanka: International Water Management Institute.

Cayuela L, JM Rey-Benayas, C Echeverría. 2006. Clearance and fragmentation of tropical montane forests in the Highlands of Chiapas, Mexico (1975-2000). Forest Ecology and Management 226: 208-218.

Chang C, C Kendall, S Silva, W Battaglin, D Campbell. 2002. Nitrate stable isotopes: tools for determining nitrate sources among different land uses in the Mississippi River Basin. Can. J. Fish. Aquat. Sci. 59: 1874-1885.

Clay A, C Bradley, A Gerrard, M Leng. 2004. Using stable isotopes of water to infer wetland hydrological dynamics. Hydrology and Earth System Sciences 8(6): 1164-1173.

Collard S, B Zammit. 2006. Effects of land-use intensification on soil carbon and ecosystem services in Brigalow (Acacia harpophylla) landscapes of southeast Queensland, Australia. Agriculture, Ecosystems and Environment (en prensa).

Cooper L, M DeNiro. 1989. Covariance of oxygen and hydrogen isotopic compositions in plant water: Species effects. Ecology 70(6): 1619-1628.

Croke A, W Merritt, A Jakeman. 2004. A dynamic model for predicting hydrologic response to land cover changes in gauged and ungauged catchments. Journal of Hydrology 291: 115-131.

De Vries J. 2000. Environmental isotopes in the hydrological cycle principles and applications. Vol. I: Introduction Theory, Methods. Willem G Mook ed. Centre for Isotope Research, Groningen, 271 p.

Echeverría C, D Coomes, J Salas, JM Rey-Benayas, A Lara, A Newton. 2006. Rapid deforestation and fragmentation of Chilean Temperate Forest. Biological Conservation 130: 481-494. 
Ehleringer J, D Bowling, L Flanagan, J Fessenden, B Helliker, L Martinelli, J Ometto. 2002. Stable isotopes and carbon cycle processes in forests and grasslands. Plant Biol. 4: 181-189.

Farley K, E Jobba, Z Gyw, R Jackson. 2005. Effects of afforestation on water yield: a global synthesis with implications for policy. Global Change Biology 11: 1565-1576.

Gremillion P, A Gonyeau, M Wanielista. 2000. Application of alternative hydrograph separation models to detect changes in flow paths in a watershed undergoing urban development. Hydrological Processes. 14: 1485-1501.

Gremillion P, M Wanielista. 2000. Effects of evaporative enrichment on the stable isotope hydrology of a central Florida (USA) river. Hydrological Processes 14: 1465-1484.

Gibson J, P Aggarwal, J Hogan, C Kendall, L Martinelli, W Stichler, D Rank, I Goni, M Choudhry, J Gat, S Bhattacharya, A Sugimoto, B Fekete, A Pietroniro, T Maurer, H Panarello, D Stone, P Seyler, L Maurice-Bourgoin, A Herczeg. 2002. Isotope studies in large river basins: a new global research focus. $\operatorname{EOS} 83$ (52)24: 613-617.

Hatchell G, C Ralston, R Foil. 1970. Soil disturbances in logging. Journal of Forestry 68: 772-775.

Henderson-Sellers A, K McGuffie, D Noone, P Irannejad. 2004. Using stable water isotopes to evaluate basin-scale simulations of surface water budgets. Journal of Hydrometeorology 5: 805-822.

Hoffmann G. 2003. Taking the pulse of the tropical water cycle. Science 3001: 776-777.

Huber A, P Barriga, R Trecaman. 1998. Efecto de la densidad de plantaciones de Eucalyptus nitens sobre el balance hídrico en la zona de Collipulli, IX Región (Chile). Bosque 19(1): 61-69.

Huber A, R Trecaman. 2004. Eficiencia del uso del agua en plantaciones de Pinus radiata en Chile. Bosque 25(3): 33-43.

Iroumé A. 1990. Assessment of runoff and suspended sediment yield in a partially forested catchment in Southern Chile. Water Resources Research 26(11): 2637-2642.

Iroumé A, A Huber, K Schulz. 2005. Summer flows in experimental catchments with different forest covers, Chile. Journal of Hydrology 300: 300-313.

Iroumé A, O Mayen, A Huber. 2006. Runoff and peak flow responses to timber harvest and forest age in southern Chile. Hydrological Processes 20: 37-50.

Kendall C, E Caldwell. 1998. Fundamentals of isotope geochemistry. In Kendall $\mathrm{C}$ and $\mathrm{JJ}$ McDonnell eds. Isotope tracers in catchment hydrology. Elsevier Science, Amsterdam. p. 51-86.

Lake J, R McKinney, F Osterman, R Pruell, J Kiddon, S Ryba, A Libby. 2001. Stable nitrogen isotopes as indicators of anthropogenic activities in small freshwater systems. Can. J. Fish. Aquat. Sci. 58: 870-878.

Liu Y, S An, Z Deng, N Fan, H Yang, Z Wang, Y Zhi, C Zhou, $S$ Liu. 2006. Effects of vegetation patterns on yields of the surface and subsurface waters in the Heishui Alpine Valley in west China Hydrol. Earth Syst. Sci. Discuss. 3: 1021-1043.

Oyarzún C, L Peña. 1995. Soil erosion and overland flow in forested areas with pine plantations at coastal mountain range, Central Chile. Hydrological Processes 9: 111-118.

Oyarzún C, H Campos, A Huber. 1997. Exportación de nutrientes en microcuencas con distinto uso del suelo en el sur de Chile (Lago Rupanco, X Región). Revista Chilena de Historia Natural 70: 507-519.

Oyarzún C, A Huber. 2003. Nitrogen export from forested and agricultural watersheds of Southern Chile. Gayana Bot. 60(1): 63-68.

Phillips F, J Hogan, S Mills, J Hendricks. 2003. Environmental tracers applied to quantifying causes of salinity in arid-region rivers: Preliminary results from the Rio Grande, southwestern USA. In Water Resource Perspectives: Evaluation, Management, and Policy. Alsharhan AS and WW Wood eds. Elsevier Science. Amsterdam. p. 327-334.

Poage M, C Chamberlain. 2001. Empirical relationships between elevation and the stable isotope composition of precipitation and surface waters: Considerations for studies of paleoelevation change. American Journal of Science 301: 1-15.

Roden J, J Ehleringer. 1999. Observations of hydrogen and oxygen isotopes in leaf water confirm the Craig-Gordon Model under Wide-Ranging Environmental Conditions. Plant Physiology 120: 1165-1173.

Squeo F, J Ehleringer. 2004. Isótopos estables: Una herramienta común para la ecofisiología vegetal y animal. In Fisiología Ecológica en Plantas: Mecanismos y Respuestas a Estrés en los Ecosistemas. Marino H ed. Ediciones de la Universidad Católica de Valparaíso, Valparaíso, Chile. p. 59-80.

Vuille M, M Werner. 2005. Stable isotopes in precipitation recording South American summer monsoon and ENSO variability: observations and model results. Climate Dynamics 25: 401-413.

Ward A, W Trimble. 2004. Environmental hydrology. $2^{\text {nd }}$ ed. Lewis Publishers. Boca Raton, Florida, USA. 475 p.

White W. 2005. Geochemistry. Chapter 9: Stable Isotope Geochemistry. Cornell University. Disponible en http://www. geo.cornell.edu/geology/classes/geo455/ Chapters.html.

Wright J, A Flecker. 2004. Deforesting the riverscape: the effects of wood on fish diversity in a Venezuelan piedmont stream. Biological Conservation 120: 439-447. 